\title{
EL “DARWINISMO PURO" DE ALFRED RUSSEL WALLACE: APORTACIONES A LA TEORÍA EVOLUTIVA MODERNA
}

\author{
Juan Manuel Rodríguez Caso \\ Facultad de Filosofía y Letras, Colegio de Historia, UNAM \\ Email: carcharhinus_7@yahoo.com \\ ORCID iD: https://orcid.org/0000-0003-0745-4657
}

Recibido: 13 febrero 2019; Aceptado: 19 junio 2020

Cómo citar este artículo/Citation: Rodríguez Caso, Juan Manuel (2020) "El "darwinismo puro" de Alfred Russel Wallace: aportaciones a la teoría evolutiva moderna", Asclepio, 72(2): p324. https://doi.org/10.3989/asclepio.2020.25.

RESUMEN: La historia suele ser insistente en recordar el caso de Alfred Russel Wallace como quien, de manera secundaria, apoyó la propuesta de Darwin. Para efectos de este trabajo se presenta lo que Wallace denominó en su obra Darwinism (1889) los elementos básicos del darwinismo puro, que servirían de base para lo que George John Romanes llamaría neodarwinismo, a partir tanto del trabajo de Wallace como del de August Weismann. Esos elementos abarcan ideas que comúnmente se asocian de manera exclusiva con el trabajo de Charles Darwin, como el concepto biológico de especie, los diferentes tipos de variación y su origen, la importancia de la selección natural como el mecanismo preponderante para entender la evolución, el rechazo a los mecanismos lamarckianos, entre otros puntos. A partir de lo anterior, los objetivos de este trabajo son dos: por un lado, rescatar esos conceptos básicos del darwinismo puro de Wallace; y por el otro, establecer algunas posibles explicaciones sobre por qué persiste la idea de que el trabajo de Wallace no parece haber sido de importancia para el desarrollo de la Síntesis Moderna.

PALABRAS CLAVE: Alfred Russel Wallace; Charles Darwin; Darwinismo; Neodarwinismo; Selección Natural.

\section{ALFRED RUSSEL WALLACE'S 'PURE DARWINISM’: CONTRIBUTIONS TO MODERN EVOLUTIONARY THEO-} RY

ABSTRACT: History tends to insist on remembering the case of Alfred Russel Wallace as one who, secondarily, supported Darwin's proposal. For the purposes of this work it is presented what Wallace called in his work Darwinism (1889) the basic elements of 'pure Darwinism', which would serve as the basis for what George John Romanes would call Neodarwinism, based on both Wallace's and August Weismann's work. These elements include ideas that are commonly associated exclusively with Charles Darwin's work, such as the biological concept of species, the different types of variation and their origin, the importance of natural selection as the preponderant mechanism to understand evolution, the rejection of Lamarckian mechanisms, among other points. From the above, the aims of this work are twofold: on the one hand, to rescue those basic concepts from Wallace's pure Darwinism; and on the other, to establish some possible explanations as to why the idea persists that Wallace's work does not seem to have been of importance for the development of Modern Synthesis.

KEY WORDS: Alfred Russel Wallace; Charles Darwin; Darwinism; Neodarwinism; Natural Selection.

Copyright: @ 2020 CSIC. Este es un artículo de acceso abierto distribuido bajo los términos de la licencia de uso y distribución Creative Commons Reconocimiento 4.0 Internacional (CC BY 4.0) 


\section{INTRODUCCIÓN}

Uno de los más importantes biólogos del siglo XX, Ernst Mayr (1904-2005), resolvía así la pregunta sobre el impacto de la teoría de la evolución a partir de las ideas de Charles Darwin (1809-1882) dentro de la biología moderna, respecto a la diversidad de maneras de llamar a la teoría evolutiva moderna: "En realidad, la mejor solución sería volver a llamarlo simplemente darwinismo. De hecho, es esencialmente la teoría original de Darwin con una teoría válida de la especiación y sin herencia blanda" (Mayr, 2004, p. 129). Sorprende, en parte, que su reconstrucción histórica no incluye a Alfred Russel Wallace (1823-1913), por lo menos en la medida en que fue el codescubridor, o co-originador, de la teoría de la selección natural, en sus propios términos, la base de la teoría moderna de la evolución.

La cercanía entre Darwin y Wallace, en cuanto a la propuesta conjunta de la teoría de la selección natural en julio de 1858, suele traer como consecuencia que se asuma que ambos autores defendieron desde el principio una misma teoría, al menos en términos generales. Bajo la presunción de que se debe hablar de un solo darwinismo, es decir, un solo conjunto de ideas atribuible en este caso a Darwin, se ha llegado incluso a afirmar la validez única de la propuesta de Darwin por sobre la de Wallace, al afirmar que "la teoría de la selección natural no estaba realmente en el paper de Wallace [el ensayo Ternate de 1858]" (Caponi, 2016, p. 68. Cursivas como en el original). Recientemente se ha afirmado que, a partir de una "reconstrucción racional de la teoría de la selección natural", las propuestas tanto de Darwin como de Wallace son diferentes, que es la capacidad explicativa y unificadora de la visión de Darwin la que debe de prevalecer, al punto de conceder que el carácter "revolucionario" de la teoría de selección natural es mérito de la propuesta de Darwin (Ginnobili y Blanco, 2019). Estas afirmaciones tienen sentido únicamente bajo la premisa de una visión anacrónica, en la cual se privilegia el desarrollo de una idea (o conjunto de ideas) por sobre otra, sin tomar en cuenta el contexto histórico en el que se desarrollaron los eventos, o que, como se verá más adelante, excluyen evidencias que resaltan la importancia de Wallace y su teoría, por escasas que puedan parecer. Incluso, propuestas históricas como la de Peter Bowler en su libro Darwin Deleted (2013), desde el marco del contrafactualismo histórico, considera que la propuesta de Darwin precede cronológicamente todo lo hecho por el propio
Wallace, para concluir que, a pesar de seguir líneas similares, este último no habría conseguido llegar nunca a una propuesta realmente como la original de Darwin. Sin embargo, aun en este último caso, Bowler se remite a una visión sesgada de la época victoriana, y sobre todo del trabajo de Wallace, que puede deberse a que su trabajo no contempla en un sentido amplio todas las fuentes disponibles, como los diarios y la correspondencia, no solamente de Darwin, sino de otros personajes de la época.

Lo anterior contrasta radicalmente con lo dicho por historiadores como M.J.S. Hodge (2015), quien se pregunta qué fue lo que distinguió tanto a Wallace como a Darwin en su época para ser ambos catalogados como "los dos primeros darwinistas", una idea que veremos más adelante, fue concedida a Wallace como resultado de su defensa del "darwinismo". Aun cuando son varios los elementos que tienen en común ambas propuestas, el carácter darwinista de ambos autores estaría en haber recuperado la obra de Charles Lyell, y trasladar las discusiones de la geología a las ciencias de la vida.

La propuesta inicial de la teoría de la selección natural, en la que por circunstancias ya discutidas ampliamente en el pasado (Himmelfarb, 1996 [1959], pp. 242-254; Beddall, 1968; Desmond y Moore, 2009) se acordó la presentación conjunta de las ideas tanto de Darwin como de Wallace, ha traído consigo una discusión estéril sobre la relación entre ambos conjuntos de ideas. El análisis de la obra de ambos autores es claro que revela dos perspectivas diferentes que buscaban explicar la transmutación de las especies, a veces a partir de ejemplos similares y otras veces con énfasis en temáticas totalmente diferentes. Afirmar la existencia de un solo conjunto de ideas denominado darwinismo, es de poca utilidad en términos históricos, una cuestión que se verá más adelante con mayor detalle. Lo que aquí se va a señalar tiene más que ver con una perspectiva que surge del propio trabajo de un autor como Wallace, y lo que en sus palabras debería de ser lo que él mismo va a denominar darwinismo. Otra parte de la discusión tiene que ver con las interpretaciones historiográficas, que tradicionalmente han brindado un lugar preponderante a Darwin por sobre cualquier otro autor al momento de reconstruir la historia de la biología, y en particular del desarrollo de la teoría de la evolución, una situación que incluso autores que han promovido la llamada "industria Darwin" lamentan, como el caso del historiador de la Universidad de 
Chicago, Robert J. Richards, o el historiador y filósofo de la ciencia de la Universidad de Florida, Michael Ruse (Richards en Cahan, 2003; Ruse, 2005).

A diferencia de la interpretación tradicional que ubica a Wallace como un mero actor secundario en el desarrollo de la teoría de la evolución, situación derivada de sus intereses "extra-cientificos", la intención en este trabajo es reconocer con mayor detalle las aportaciones de su obra al desarrollo teórico de la teoría de la evolución. La cuestión es valorar que conceptos e ideas clave de la biología evolutiva del siglo XX parecen tener más que ver con lo dicho por Wallace que con Darwin, por lo menos en la medida en que el primero defendió a ultranza cuestiones como el gradualismo extremo o la omnipotencia de la selección natural, todo ello recuperado en su momento por la Síntesis Moderna. Lo que se busca es contribuir a comprender la pluralidad y complejidad en el desarrollo de la práctica científica, y en particular, de la construcción de la teoría evolutiva.

Derivado de lo anterior, este trabajo presenta en primera instancia una reflexión histórica sobre el propio término darwinismo, para mostrar con ello la polisemia del término a lo largo del siglo XIX, pero, sobre todo, el uso que el mismo Darwin le dio a lo largo de su obra, que como veremos, no contribuye necesariamente a dilucidar de manera clara cuál podría ser su significado apropiado. Posteriormente, se describe lo que Wallace denominó darwinismo puro en su obra Darwinism (1889), ya que fue ahí donde planteó los conceptos básicos que posteriormente serían asociados con la visión más tradicional del neodarwinismo. Finalmente, se discute la relación de las ideas de Wallace con las propuestas concretas desarrolladas como parte de las discusiones modernas sobre evolución, que usualmente se asocian exclusivamente con la propuesta de Darwin.

\section{2. ¿EXISTE SOLAMENTE UN DARWINISMO?}

Puede resultar extraño en principio hablar de que no existe un solo darwinismo, aunque inicialmente habríamos de pensar a qué se hace referencia realmente cuando se menciona tal término. Una búsqueda a nivel de diccionario nos remite a valorar los cambios que sufrió el término a lo largo del siglo XIX, por lo menos en el inglés: un primer uso sería el que dio el médico Benjamin Ward Richardson (1828-1896) en 1856 para referirse a la postura del abuelo de
Charles, Erasmus Darwin (1731-1802), como "la hipótesis del desarrollo de los seres vivos desde un centro primordial". En 1864, el zoólogo Thomas H. Huxley (1825-1895) consideraba que darwinismo se refería a una posición filosófica, que ponía a la evolución como eje central en un sentido antiteleológico, todo esto como parte de una serie de críticas del anatomista suizo Rudolph Albert von Kölliker (18171905), que afirmaba que Darwin era un teleólogo. Otra mención fue en 1871 en la influyente revista literaria The Athenaeum, en una reseña de On the Genesis of Species, del anatomista George Jackson Mivart (1827-1900), quien proponía, entre otras cosas, una reconciliación entre el catolicismo y la evolución, a lo que el autor de la reseña responde: "La verdad es que es imposible reconciliar a los Doctores de la Iglesia con los Doctores del Darwinismo". En la traducción que hizo el naturalista británico Edwin Ray Lankester (1847-1929) de The history of creation, or, The development of the earth and its inhabitants by the action of natural causes: doctrine of evolution in general, and of that of Darwin, Goethe, and Lamarck in particular (1876) del naturalista y filósofo alemán Ernst Haeckel (1834-1919), se plantea en la introducción que el darwinismo es un fragmento de una doctrina mucho más amplia que tiene como objetivo explicar el desarrollo, o transformación, natural de los organismos. La última definición corresponde a la planteada por Wallace en su obra de 1889 , que se expondrá con mayor detalle más adelante ${ }^{1}$.

Ernst Mayr dedicó buena parte de su labor como historiador y filósofo de la biología a la defensa del papel de Darwin como el originador del pensamiento evolutivo moderno, y en ese sentido, a reflexionar sobre la importancia de las ideas de Darwin para la consolidación de la biología del siglo XX. Su recuento sobre lo que en su opinión era el darwinismo, empieza por una afirmación tajante sobre el carácter no monolítico de la teoría evolutiva, que concede que en todo caso inició con el propio Darwin (Mayr, 1991, p. 90), quien se refirió a su propuesta desde la primera edición de On the Origins como la "teoría de la descendencia con modificación mediante la selección natural" (Darwin, 1859, p. 459), una idea que como se verá más adelante, será retomada profusamente por Wallace, en una postura que mostraba que "estaba muy adelantado a su tiempo en su campaña por la selección natural" (Mayr, 1991, p. 92).

El listado que proporciona Mayr abarca interpretaciones de lo más variadas: darwinismo como la "teo- 
ría de la evolución de Darwin"; darwinismo como evolucionismo; darwinismo como anticreacionismo; darwinismo como anti-ideología; darwinismo como seleccionismo; darwinismo como evolución variacional; darwinismo como el credo de los darwinistas; darwinismo como una nueva cosmovisión; y darwinismo como una nueva metodología (Mayr, 1991, pp. 92-106). A pesar de tal pluralidad, que incluso lleva a Mayr a afirmar que la mayoría de estos significados "son claramente engañosos o no representativos del pensamiento de Darwin" (Mayr, 1991, p. 106), concluye que hay dos que se distinguen por su amplia aceptación: de mediados del siglo XIX hasta inicios del XX, darwinismo como la manera de explicar los procesos naturales del mundo vivo; y en un sentido "moderno", como "cambio evolutivo adaptativo bajo la influencia de la selección natural, y evolución variacional en lugar de transformacional" (Mayr, 1991, p. 107). Vale la pena recordar aquí, que, aunque la reconstrucción de Mayr se plantea en términos históricos, como se ve en la introducción de este trabajo, para él hay una definición que debe ser defendida por los autores modernos, para evitar con ello malas interpretaciones. Sin embargo, aun con las mejores intenciones, si se parte de reconocer el pluralismo de Darwin, es claro que decantarse por algún elemento en particular de su explicación evolutiva parte de los intereses y sesgos de quien lo esté leyendo.

Después de estas interpretaciones, cabe preguntarse qué se discutía en términos amplios durante los tiempos de Darwin mismo. El historiador británico James Moore nos recuerda que hablar de darwinismo en el siglo XIX es pensar en un "término cargado" (Moore, 1991, p. 355), un punto de coincidencia con lo dicho por Mayr, pero el análisis de este último no proporciona a veces muchos detalles de lo que se discutía en la época ${ }^{2}$. La propuesta de Moore nos remite a muchas de las discusiones que se dieron a lo largo de la década de 1860 , de particular importancia si se considera que ese periodo se ubica entre las dos publicaciones más conocidas de Darwin, El origen de las especies (1859) y El origen del hombre (1871).

Resulta particular ver que las ocasiones en que el propio Darwin se refiere al término "darwinismo" es para hablar de lo que otros autores entienden. En su obra escrita, como en el primer volumen de The Descent of Man (1871), las únicas referencias al término son notas al pie a obras que en su mayoría eran críticas ("Anti-darwinism", del reverendo James McCann, presentada el 20 de agosto de 1869 en la reunión de la British Association for the Advancement of Science (BAAS) en Exeter; "Darwinism and National Life", publicado en Nature el 16 de diciembre de 1869 por Ladies' Educational Association de Londres), o aplicaciones de su propuesta ("Philology and Darwinism" publicado en Nature el 24 de marzo de 1870 por el reverendo Frederic William Farrar (1831-1903)); la traducción al inglés Darwinism tested by the Science of Language (1869), del lingüista alemán August Schleicher (1821-1868); en el segundo volumen también se encuentra una mención a un artículo escrito por el botánico alemán Matthias Jakob Schleiden (1804-1881), "Ueber des Darwinismus und die damit zusammenhängenden Lehren" ["Sobre el darwinismo y sus enseñanzas relacionadas"], publicado en 1869 en el periódico Unsere Zeit. En la segunda edición incluyó referencias como: "Darwinism and Divinity, Essays on Free-thinking", un ensayo del historiador y editor del Oxford English Dictionary, Leslie Stephen (1832-1904), publicado en Essays on Freethinking and Plainspeaking (1873), que planteaba la existencia de límites entre las capacidades mentales de seres humanos y animales; Darwinism in Morals, and Other Essays (1872), de la líder sufragista irlandesa Frances Power Cobbe (1822-1904); Le Darwinisme et L'Origine de L'Homme (1873), del padre Alphonse Joseph Lecomte (1845-1927); "Ueber der Tonapparat der Locustiden, ein Beitrag zum Darwinismus" ["Sobre el aparato de sonido de los lóbulos, una contribución al darwinismo"], publicado en 1872 en Zeitschrift für wissenschaftliche Zoologie [Revista de Zoología Científica] por el entomólogo austríaco Vitus Graber (1844-1892).

En primera instancia, es claro que, a partir de estas evidencias, el propio Darwin no habló en ningún momento de un conjunto de ideas denominado como darwinismo, o incluso, no reconoció explícitamente a qué se refería el término como tal a lo largo de su obra. Más bien, parece dar por sentado que es la manera en que otros autores se referían a su obra en general, es decir, a las interpretaciones y alcances que se podían asociar con sus dichos. Vale recordar que Darwin se distinguió a lo largo de su obra por "su retórica maravillosamente ambigua" (Kohn, 1989, p. 215), que en ocasiones se veía reflejada en esa falta de claridad conceptual. Pero, al mismo tiempo, es una muestra de la enorme diversidad de formas de entender el impacto de las ideas de Darwin, que se puede resumir así: 
Desde el "darwinismo puro" como lo que Darwin enseñó originalmente (Wallace, Gray), hasta el "darwinismo" como lo que Darwin realmente enseñó (Romanes, Hodge), o lo que su abuelo enseñó y que él mismo debería haber enseñado (Butler), al "verdadero darwinismo" como lo que Darwin habría enseñado si se le hubiera presentado la oportunidad (Henslow), y finalmente, al "darwinismo puro" como lo que Darwin enfáticamente no enseñó (Driesch) las opciones hermenéuticas son lo suficientemente desordenadas como para hacer que un erudito ordenado quiera tomar partido. Después de todo, ¿quién tiene razón? ¿Quién interpretó correctamente lo que dijo Darwin? ¿Quién entendió lo que Darwin realmente quería decir? ¿Quién tiene derecho a representar al auténtico darwinismo? (Moore, 1991, p. 358. Cursivas como en el original)

Esto se puede complementar con una breve descripción de algunos de esos diferentes darwinismos, que permite valorar así los alcances de la propuesta de Darwin. Una de las más arduas críticas vino del teólogo presbiteriano estadounidense Charles Hodge (1797-1878), una propuesta cuya importancia reside en la profunda influencia que mantiene entre el protestantismo más conservador (Marsden, 2006, p. 227). Dentro de la larga exposición que hace Hodge en el libro, hay un resumen que deja clara su posición: “¿Qué es el darwinismo? Es Ateísmo. Esto no significa, [...] que el Sr. Darwin mismo y todos los que adoptan sus puntos de vista son ateos; pero significa que su teoría es atea; que la exclusión del diseño de la naturaleza es, como dice el Dr. Gray, equivalente al ateísmo" (Hodge, 1874, p. 177). La preocupación de Hodge estaba en la defensa de la visión propuesta por William Paley (1743-1805) en contra del papel ciego que se buscaba otorgar a la selección natural como explicación de las causas naturales, argumento sustentado en buscar desde la ciencia encontrar nuevas maneras de interpretar las Escrituras, una situación que no necesariamente lo alejaba de su firme convicción calvinista sobre el papel absolutamente soberano de Dios (Gutjahr, 2011, p. 369-371).

Uno de los casos más llamativos que se pueden mencionar aquí, es la discusión que sostuvieron el propio Wallace y el fisiólogo canadiense George John Romanes (1848-1894), "el principal discípulo de Darwin" (Elsdon-Baker, 2008, p. 75), en la que puede ser la disputa más áspera que en términos intelectuales tuvo el propio Wallace a lo largo de su vida, incluyendo las numerosas diferencias con Darwin ${ }^{3}$.
Poco tiempo después de la publicación de Darwinism, Romanes afirmaba que la doctrina de Darwin no implicó en ningún momento la defensa a ultranza de la selección natural como la única causa de la evolución orgánica, a la par de reconocer la importancia de lo que él denominaba "la teoría fisiológica de la selección" (Romanes, 1889a, p. 645). Poco tiempo después, en una reseña mucho más amplia, Romanes llevó la crítica a Wallace mucho más lejos. A expensas de resaltar la enorme importancia de la obra de Darwin, Romanes deja una afirmación que permite apreciar la eterna discusión sobre Wallace y Darwin: "Es notorio que, desde el momento en que publicaron su teoría conjunta de la evolución por selección natural, Darwin y Wallace no lograron ponerse de acuerdo sobre ciertos puntos de la doctrina que, aunque de importancia comparativamente pequeña en relación con cualquier cuestión de evolución considerada como un hecho, eran, y siguen siendo, de la mayor importancia posible en relación con la cuestión de la evolución considerada como un método" (Romanes, $1889 b$, p. 151). En términos generales, y de manera análoga a lo que el propio Wallace hacía en Darwinism, Romanes hacia suyas las palabras de Darwin para establecer las diferencias entre ambos autores en temas como la selección sexual, la herencia de caracteres adquiridos, y la esterilidad de especies, para concluir que, salvo la manera en la que Darwin abordó esos temas de manera adecuada, “[...] me parece que el último trabajo del Sr. Wallace es uno de los más interesantes y sugestivos de toda la literatura darwiniana" (Romanes, 1889b, p. 155). Al momento de valorar esta crítica no hay que olvidar que Romanes veía a Darwin en términos básicamente religiosos, era un auténtico converso a su doctrina (Pleins, 2014, pp. x-xii), situación que se puede valorar ampliamente a partir del poema que Romanes escribió, Charles Darwin: A Memorial Poem (Pleins, 2014, pp. 299-348).

El planteamiento de Hans Adolf Eduard Driesch (1867-1941) en The Science and Philosophy of the Organism: The Gifford Lectures delivered before the University of Aberdeen in the Year 1907 and 1908 $(1908)^{4}$, fue por decir lo menos, extravagante. Driesch fue un filósofo y biólogo prusiano, ampliamente reconocido por sus trabajos sobre embriología y por su defensa de la filosofía neovitalista de la entelequia. La definición inicial que encontramos en la obra deja clara una visión de Darwin y sus ideas diametralmente diferente a lo dicho por otros autores: "Darwin, el mismo tipo de hombre dedicado sólo a la ciencia y 
no a los intereses personales, Darwin era todo menos dogmático, y sin embargo el darwinismo es el dogmatismo en una de sus formas más puras" (Driesch, 1908, p. 260). Se concede que la fuerza de su razonamiento estuvo en conceder un papel vital a las variaciones y la selección natural, pero, además: "Era lamarckiano hasta cierto punto. Y no tenía una opinión definitiva sobre el origen y la naturaleza más íntima de la vida en general. Estos pueden parecer defectos, pero en realidad son ventajas de su teoría" (Driesch, 1908, p. 260). Driesch plantea así a Darwin como un auténtico hombre de ciencia, que no busca la verdad absoluta, ya que, por el contrario, son los seguidores de Darwin quienes, en su opinión, terminaron por remover las enseñanzas de Darwin del darwinismo. De hecho, Driesch no solamente va a considerar al darwinismo como una teoría incompleta - al menos en lo que él denomina su versión dogmática - sino también al lamarckismo, ya que considera que la única manera de entender la transformación de las especies es a través del estudio de la filogenia de los individuos (Driesch, 1908, p. 304-305), en una clara defensa de los estudios embriológicos.

El último ejemplo para considerar también tiene una parte que ronda la ironía y el morbo. El autor de la crítica fue el clérigo anglicano y botánico George Henslow (1835-1925), quien fue el tercer hijo del Reverendo John Stevens Henslow (1796-1861), ampliamente conocido por haber sido mentor de Darwin en Cambridge, y quien le consiguió un lugar en el famoso viaje del Beagle (1831-1836). Resultado de una conferencia presentada ante la Royal Horticultural Society el 5 de marzo de 1907, se publicó en la revista de la sociedad "The True Darwinism" en ese mismo año. Su primera aproximación fue hablar del darwinismo como una derivación lógica de la obra de Darwin publicada en 1859, con énfasis en las variaciones y en la selección natural, pero, Henslow recordaba que el propio Darwin reconoció que había otros medios para explicar la adaptación de los organismos, y es a partir de ahí que recuperó "la versión lamarckiana" de Darwin, para justificar que, a partir de la evidencia experimental en plantas que se acumuló a lo largo del siglo XIX, el "darwinismo verdadero" - en contraposición del "nuevo darwinismo" - surgía de la siguiente conclusión: "Es que el darwinismo, o 'El origen de las especies por medio de la selección natural', debe ser reemplazado por 'El origen de las especies por medio de la respuesta con adaptación a la acción directa de las nuevas condiciones de vida'. Esta es la base real de la Evolución" (Henslow, 1907, p. 7).
Este no es más que un muy breve listado de las posiciones que surgieron como parte de las discusiones alrededor del darwinismo, y es que, en la práctica, es un trabajo pendiente de los historiadores valorar adecuadamente muchas de las obras que, sobre todo, criticaban la obra asociada con Darwin ${ }^{5}$.

La discusión se puede llevar incluso a la manera en la que Wallace concedía la importancia de Darwin al momento de hablar de su teoría conjunta, y a la percepción de sus contemporáneos sobre su lugar dentro de las discusiones sobre la evolución de las especies. El conocido escritor británico Samuel Butler (18351902), un arduo crítico de la teoría de la selección natural, decía lo siguiente, como parte de su análisis del darwinismo decimonónico:

Pasemos ahora al exponente más autorizado de la evolución de los últimos días - me refiero al Sr. Wallace, cuya obra, titulada "Darwinismo", aunque debería haberse titulado "Wallaceísmo", sigue siendo tan darwinista que desarrolla la enseñanza del Sr. Darwin en la dirección que le dio el propio Sr. Darwin. (Butler, 1908, p. 236)

En esa misma línea de diferenciar las propuestas de Darwin y Wallace, hubo dichos similares con el paso de los años. El naturalista británico Frederick Wollaston Hutton (1836-1905) comentaba en una breve nota en Science que, aunque efectivamente la propuesta de Wallace y de August Weismann (1834-1914) daba protagonismo a la selección natural al mismo tiempo que se rechazaba la herencia de caracteres adquiridos, lo más apropiado era llamar a esa propuesta nuevo darwinismo, ya que algunas de las ideas propuestas por ambos autores "estaban en desacuerdo con las que siempre tuvo Darwin hasta su muerte" (Hutton, 1900, pp. 588-589). De hecho, la propuesta de Hutton es que la propuesta de Wallace se denominara como wallaceísmo, para diferenciarla de la visión original de Darwin. En un sentido similar, el zoólogo holandés Ambrosius Arnold Willem Hubrecht (1853-1915) declaraba en un artículo en Contemporary Review de noviembre de 1908 , que, desde su punto de vista, la propuesta original de Darwin, el darwinismo, hacía hincapié en el papel de las variaciones - ya que sin ellas no podría funcionar la selección natural -, a partir sobre todo de trabajos como los de Hugo de Vries, lo que confirmaba las intuiciones iniciales de Darwin, mientras que la propuesta de Wallace, con ese marcado énfasis en la selección natural, es a lo que se debería llamar wallaceísmo (Hubrecht, 1908, pp. 632634; Fichman, 2004, p. 310). 
¿Y cuál era la opinión de Wallace en toda esta discusión? La única respuesta fue a los comentarios de Hubrecht, en el mismo Contemporary Review, en donde en una pequeña nota afirmaba que no había ninguna divergencia entre su visión y la de Darwin, al recalcar que ambos defendían la importancia de las variaciones individuales, en contraposición con los "sports", posición defendida por el propio Hubrecht y otros mutacionistas. Esta frase deja clara su posición: "Es sólo la inmensa acumulación de hechos durante el último cuarto de siglo, tanto en cuanto a la cantidad y universalidad de las "variaciones individuales", como en cuanto a la extrema rigidez de la "selección natural", lo que ha llevado a la mayoría de los darwinianos y a mí mismo a dar un paso más allá de lo que Darwin fue capaz de hacer, y a dudar si alguna vez se han originado "variaciones individuales" en una "especie" natural" (Wallace, 1908, p. 717). Es posible que, ese continuo afán de poner a Darwin por delante, y colocarse a sí mismo como su defensor e intérprete, hiciera muy complicado tener mayor protagonismo. Y es que, como se verá a continuación, hubo razones prácticas que llevaron a Wallace a defender su darwinismo.

\section{EL "DARWINISMO PURO": PROPUESTAS GENERALES DE UN DEFENSOR DEL DARWINISMO}

Una de las observaciones que hace Wallace en su autobiografía respecto al proceso de escritura de Darwinism, tiene que ver con las razones que lo llevaron a considerar llevar a un público más amplio las conferencias que por esos años, entre 1886 y 1887, había presentado a lo largo de Canadá y Estados Unidos. La opción del viaje surgió de las pláticas que tuvo con el paleontólogo estadounidense Othniel Charles Marsh (1831-1899) durante la visita que realizó este último a Inglaterra a finales de la década de 1870 (Wallace, 2013a, p. 6), y de las que sacó la siguiente conclusión:

Muchos de mis corresponsales, así como personas que conocí en América, me dijeron que no podían entender el "Origen de las Especies" de Darwin, pero sí mi conferencia sobre el "Darwinismo"; y por lo tanto, se me ocurrió que una exposición popular sobre el tema podría ser útil, no sólo para que el lector en general pudiera entender a Darwin, sino también para que sirviera como respuesta a los muchos artí- culos y libros que profesan refutar la teoría de la selección natural. (Wallace, 1905, vol. II, p. 201).

Esas conferencias fueron patrocinadas por el Lowell Institute de Boston, Massachusetts, una fundación con fines educativos fundada en 1839 a partir del apoyo financiero del filántropo estadounidense John Lowell, Jr. (1799-1836). Como parte de los esfuerzos por ampliar los alcances de la educación, se propuso la existencia de espacios en que se "destacaran las "conferencias-demostraciones" nocturnas sobre "ciencias prácticas" como medio para llegar al obrero" (Rossiter, 1971, pp. 602-603). Si se considera este último en relación con lo dicho por Wallace en su autobiografía, es claro que buena parte del público no contaba posiblemente con los elementos necesarios para en principio entender a cabalidad la obra más conocida de Darwin. Aunque la idea de la transformación era de sobra conocida por el público británico gracias a obras como Vestiges of the Natural History of the Creation (1844) del periodista escocés Robert Chambers (1802-1871), las ideas de Darwin fueron recibidas de manera controversial - sobre todo aquellas relacionadas con el ser humano - debido a su continuo uso ideológico en la prensa:

El darwinismo era un tema muy controvertido, y se utilizaron todo tipo de argumentos en la batalla ideológica en torno a él. Uno de estos argumentos -quizás el más eficaz entre los no especialistas- era representar al propio bando como fuerte y triunfante, y al bando contrario como débil y dividido. La verdad, como la victoria, está del lado de los grandes batallones (Ellegård, 1990, p. 39).

Y es que, aunque hoy en día tenemos relatos como los del propio Mayr sobre la relevancia de las ideas de Darwin y su capacidad "revolucionaria", o los de filósofos de la ciencia que afirman que la misma propuesta de "un largo argumento" tiene que ser vista como el resultado menor de la obra que estuvo desarrollando desde que regresó del viaje del Beagle, cuya escritura había iniciado en 1856 y que estaba destinada a ser titulada Natural Selection (Hodge, 1977 , p. 237), estaba estructurado como un resumen que "no estaba en un estilo técnico ni copiosamente referenciado", centrado en dos ideas: el árbol de la vida y la selección natural (Waters, 2009, pp. 120-121).

Esto no habla necesariamente de que el escrito en sí mismo fuera de fácil lectura, o que la comprensión de los temas presentados fuera sencilla para los lec- 
tores, aun para aquellos con formación científica. De hecho, el mismo Waters señala una situación que fue común entre los lectores de la obra de Darwin:

Aunque Darwin puede haber diseñado su libro para ser leído como un largo argumento para la evolución por medio de la selección natural, muchos de sus lectores deben haberlo leído de manera diferente. Sabemos esto porque el Origen persuadió a muchos lectores a aceptar la idea de la "evolución", pero no la parte de la visión de Darwin de la "selección natural" (Waters, 2009, pp. 120).

$Y$ es que, aunque pueda sonar controversial, es posible que la intención inicial de Darwin, aun con el caso del "resumen", fuera no convencer a un público amplio sobre la realidad de la transformación de las especies, o de la importancia de mecanismos como la selección natural, sino más bien, convencer a una sola persona. Y no a cualquier persona, sino al hombre de ciencia más importante de la época victoriana, el geólogo escocés Charles Lyell (1797-1875) (Dixon y Radick, 2009, p. 26-27), una situación que se debe entender a partir de que la metodología y el enfoque que Darwin - y también Wallace - va a retomar para explicar la transformación de las especies derivó en su totalidad de la propuesta lyelliana (McKinney, 1972; Hodge, 2015).

Retomando el viaje de Wallace por Norteamérica, es importante ubicar su lugar dentro de la comunidad científica de la época, y es que a pesar de su discreción y hasta su timidez en público, la invitación del Lowell Institute servía para ratificar que: "En ese momento, tras la muerte de Darwin en 1882, era el naturalista más famoso del mundo" (Wallace, 2013a, p. 7). Una clara muestra de esa posición, y de su lugar como el auténtico defensor de las ideas de Darwin, se puede ver en la mención aparecida en el periódico Boston Evening Transcript:

El primer darwinista, Wallace, no dejó una pierna para que el anti-darwinismo se parara cuando había terminado su primera conferencia de Lowell anoche. Fue una obra maestra de declaración condensada tan clara y simple como compacta - un hermoso espécimen de trabajo científico. El Sr. Wallace, aunque no es un orador, es probable que se convierta en uno de los favoritos como conferenciante, su manera es tan genuinamente modesta y directa ${ }^{6}$.

Una perspectiva que suele perderse de vista cuando se recuerda a Wallace es que, junto a personajes como Thomas Huxley fungió un papel fundamental en la defensa y difusión de las explicaciones evolutivas. Simplemente, Darwinism fue el resultado de un viaje realizado por Estados Unidos y Canadá, cuyo objetivo principal fue, tanto aclarar qué era la propuesta de la selección natural, como defenderla de los embates y constantes críticas. Aquí, el objetivo es recuperar la propuesta que en propias palabras de Wallace sería el "darwinismo puro", a partir de una obra cuyo título resulta un tanto controversial: Darwinism (1889). La aclaración inicial puede ser reveladora:

El presente trabajo trata el problema del Origen de las Especies en las mismas líneas generales que fueron adoptadas por Darwin; pero desde el punto de vista alcanzado después de casi treinta años de discusión, con una abundancia de nuevos hechos y la defensa de muchas teorías nuevas o viejas (Wallace, 1889, v).

Una de las particularidades de la obra, que sirve además como un homenaje a Darwin y On the Origin of Species (1859), es recuperar su estructura argumental, con algunas diferencias nada sutiles. Al igual que la obra de Darwin, la primera parte la enfocó Wallace en explicar la base de la teoría: la importancia de la variabilidad, en su propias palabras: "El fundamento de la teoría darwiniana es la variabilidad de las especies, y es bastante inútil tratar de comprender esa teoría, y mucho menos apreciar la integridad de la prueba de la misma, a menos que primero obtengamos una concepción clara de la naturaleza y el alcance de esa variabilidad" (Wallace, 1889, p. 41), para así recalcar el papel de la domesticación de especies, en la lucha por la existencia y la selección natural. Sobre la importancia de la lucha por la existencia menciona:

No hay ningún fenómeno de la naturaleza que sea a la vez tan importante, tan universal y tan poco comprendido, como la lucha por la existencia que se libra continuamente entre todos los seres organizados. A la mayoría de las personas la naturaleza les parece tranquila, ordenada y pacífica. [...] Pero no ven, y casi nunca piensan, en los medios por los cuales se produce esta belleza, armonía y disfrute. No ven la búsqueda constante y cotidiana de alimento, el fracaso en la obtención que significa debilidad o muerte; el esfuerzo constante por escapar de los enemigos; la lucha siempre recurrente contra las fuerzas de la naturaleza (Wallace, 1889, p. 14).

El papel de la variación en estado doméstico se vuelve fundamental en esta propuesta, como una 
manera de incrementar las variaciones, en un proceso que se da tanto de manera consciente como inconsciente por parte del ser humano (Wallace, 1889, pp. 83-101). Un punto importante que resalta Wallace es el caso de la divergencia de carácter, que para él tiene dos propósitos:

En primer lugar, permite que una especie que está siendo superada por los rivales, o está en proceso de extinción por los enemigos, se salve adoptando nuevos hábitos u ocupando lugares vacantes en la naturaleza. Este es el efecto inmediato y obvio de todos los numerosos ejemplos de divergencia de carácter que hemos señalado. Pero hay otro resultado menos obvio, que es que cuanto mayor sea la diversidad de los organismos que habitan en un país o distrito, mayor será la cantidad total de vida que se puede mantener allí (Wallace, 1889, pp. 109-110).

Ahora, una de las diferencias más notorias de la obra es que desde el inicio expresamente define qué es una especie, algo que Darwin no hizo de manera explícita en su obra de 1859. La intención inicial de Wallace es proporcionar una base conceptual a partir de la que cualquiera pueda entender realmente los alcances de la teoría. A partir de las propuestas del botánico suizo Augustin Pyrame de Candolle (17781841) y del zoólogo británico William John Swainson (1789-1855) resalta que una especie se distingue en primera instancia por una morfología particular, y por la particularidad de cruzarse entre individuos del mismo tipo, lo que permite que los individuos se parezcan a sus progenitores (Wallace, 1889, pp. 1-2). Es importante señalar que esta idea no era nueva para Wallace, en la medida en que ya las había desarrollado en términos similares tanto en su "cuaderno de especies", escrito entre 1855 y 1859 (Wallace, 2013b), en sus estudios sobre las mariposas de la familia Papilionidæ (Wallace, 1865; Mallet, 2009), y de manera muy clara y concisa en la discusión que hace sobre lo que denomina "leyes y modos de la variación" en 1870, en donde define a la especie verdadera como: "Las especies son simplemente aquellas razas o formas locales fuertemente marcadas que cuando están en contacto no se entremezclan, y cuando habitan áreas distintas se cree generalmente que han tenido un origen separado, y que son incapaces de producir una descendencia híbrida fértil” (Wallace, 1870, p. 161).

Dado que una parte importante de los objetivos de Wallace con Darwinism era el afirmar la validez de las explicaciones seleccionistas, siempre bajo un mar- co estrictamente utilitarista, defendió a ultranza la utilidad de las adaptaciones, en lo que posteriormente se denominó como adaptacionismo. La defensa del principio de utilidad, que tantas críticas le han generado a Wallace en términos historiográficos, no es sino la extensión de lo afirmado por el propio Darwin, cuando en Origins afirma que la selección natural no puede producir ni una estructura dañina a un organismo ni una estructura que sea demasiado perfecta de acuerdo con las necesidades de un organismo en una etapa particular de su desarrollo evolutivo (Darwin, 1859, pp. 201-202; Fichman, 2004, p. 192). Para Wallace entonces, todas aquellas características que no representen utilidad para los organismos, serán inestables y consecuentemente, desparecerán por la acción de la selección natural (Wallace, 1889, pp. 138-142).

Otro aspecto importante de la explicación de Wallace fue el papel de la infertilidad entre diferentes especies: "[l]a esterilidad entre especies cercanas era resultado de la acción de la selección natural; y pensaba que los distintos grados de esterilidad eran pasos graduales hasta llegar a la esterilidad absoluta, y que esas variaciones en los niveles de esterilidad podían acumularse y ser favorecidas por la selección natural" (Darwin y Wallace, 2009, pp. 28-29). En los términos del propio Wallace:

Si aceptamos la asociación de algún grado de infertilidad, por leve que sea, como un acompañamiento no poco frecuente de las diferencias externas que siempre surgen en un estado de naturaleza entre variedades y especies incipientes, se ha demostrado que la selección natural tiene poder para aumentar esa infertilidad de la misma manera que tiene poder para aumentar otras variaciones favorables. Este aumento de la infertilidad será beneficioso siempre que surjan nuevas especies en la misma zona que la forma parental; y así vemos cómo, de las cantidades fluctuantes y muy desiguales de infertilidad correlacionadas con las variaciones físicas, puede haber surgido esa cantidad mayor y más constante que parece caracterizar normalmente a las especies bien marcadas (Wallace, 1889, p. 186).

Todo lo anterior, era sustentado en dos conjuntos de evidencias, que fueron clave tanto para Darwin como para Wallace desde su inicio como naturalistas: la geografía y la geología. Sobre esta última, vale la pena recordar lo mencionado anteriormente en este trabajo sobre la influencia clave de la obra de Charles Lyell, que, planteó ideas como el tiempo profundo y 
el gradualismo, conceptos que permitieron tanto a Darwin como a Wallace entender la naturaleza de los fósiles y la extinción, aunque uno de los problemas comunes a que se enfrentaron ambos fueron los huecos en el registro fósil. A esto último, con gran optimismo, Wallace decía que con el paso del tiempo las evidencias necesarias para llenar esos huecos se irían descubriendo (Wallace, 1889, p. 409). Por otro lado, la geografía, es posiblemente el área donde mayor reconocimiento ha tenido, de la que se le reconoce como "padre de la biogeografía", o por lo menos de la zoogeografía. Wallace proporciona con esto una síntesis de zoología, geología y evolución por selección natural, que da como resultado un marco causal a partir del cual explicar la distribución de los organismos en términos evolutivos (Fichman, 2004, p. 49). Esto permitiría la consolidación de regiones zoológicas en términos geográficos (Wallace, 1889, pp. 348349) que le permitirían explicar diferencias regionales, como las existentes entre el sur de Asia y Oceanía, lo que en su momento fue denominado como "línea de Wallace" (Fichman, 2004, p. 50)7.

Hay dos temas que destacan dentro del discurso darwiniano de Wallace: su contundente rechazo al lamarckismo y el controvertido caso de la evolución humana. Es de sobra conocido que el rechazo a los mecanismos lamarckianos fue una de las grandes diferencias entre la propuesta de Wallace y la de Darwin. Wallace consideraba que no existían suficientes evidencias en la naturaleza que pudieran explicar realmente la herencia de caracteres adquiridos, o el uso y el desuso de ciertas características que posteriormente serían heredadas, más allá de similitudes morfológicas (Wallace, 1889, pp. 411-423). Las críticas eran dirigidas particularmente al polímata británico Herbert Spencer (1820-1903) y al anatomista y paleontólogo estadounidense Edward Drinker Cope (1840-1897), como los principales difusores de esas ideas a finales del siglo XIX. Sobre la imposibilidad de la herencia de caracteres adquiridos, retoma la propuesta de Weismann para afirmar que "un resultado lógico de la teoría [de la herencia] es la imposibilidad de la transmisión de caracteres adquiridos, dado que la estructura molecular del germoplasma está ya determinada dentro del embrión" (Wallace, 1889, p. $440)$, con lo que buscaba dar una respuesta actualizada sobre las explicaciones lamarckianas.

Sobre el caso del ser humano, vale la pena resaltar que, a pesar de los diversos intereses académicos de Wallace, estudiar al ser humano en sus más variados aspectos fue el eje fundamental de su labor como naturalista (Rodríguez Caso, 2019, p. 142). El primer paso para Wallace es reconocer la "animalidad" del ser humano, en virtud de las evidencias morfológicas disponibles, que sobre todo permiten ver las continuidades ${ }^{8}$ - haciendo hincapié en los órganos rudimentarios y las variaciones en los mismos a lo largo del tiempo - entre diferentes especies animales, aunque planteando ya una distancia con la propuesta de Darwin: "El punto que quiero especialmente llamar la atención es que, probar la continuidad y el desarrollo progresivo de las facultades intelectuales y morales de los animales al hombre, no es lo mismo que probar que estas facultades hayan sido desarrolladas por selección natural" (Wallace, 1889, p. 463). Esta afirmación tiene que verse a partir de una defensa a ultranza del gradualismo como la forma de explicar el proceso evolutivo, y es que la explicación de Wallace sobre el origen y desarrollo de facultades intelectuales como la capacidad matemática, musical y artística no surgieron a partir de "condiciones rudimentarias" en grupos de "salvajes", sino que "aparecen casi de repente y en perfecto desarrollo en las razas civilizadas más avanzadas" (Wallace, 1889, p. 473). En cierta medida, Wallace abre la discusión sobre el papel que juega la cultura - o más propiamente, el avance cultural - en el desarrollo pleno de capacidades de los seres humanos. Wallace admite entonces que: "No cabe duda de que la continuidad admitida del progreso del hombre desde el bruto no admite la introducción de nuevas causas, y que no tenemos pruebas del cambio repentino de la naturaleza que tal introducción provocaría" (Wallace, 1889, p. 474), y define que el proceso evolutivo del mundo orgánico se debe entender en tres etapas: 1 ) el cambio de lo inorgánico a lo orgánico, 2) la introducción de la sensación o conciencia, y 3) la aparición de características distintivas del ser humano (Wallace, 1889, p. 474-476).

El cierre del libro, a pesar de que la intención inicial es defender las ideas de Darwin, deja clara la particular manera en la que Wallace va a entender su darwinismo:

Encontramos así que la teoría darwiniana, incluso cuando se lleva hasta su conclusión lógica extrema, no sólo no se opone a la creencia en la naturaleza espiritual del hombre, sino que le presta un apoyo decidido. Nos muestra cómo el cuerpo del hombre puede haberse desarrollado a partir del de una forma animal inferior bajo la ley de la selección natural; pero 
también nos enseña que poseemos facultades intelectuales y morales que no podrían haber sido mayormente desarrolladas, sino que deben haber tenido otro origen; y para este origen sólo podemos encontrar una causa adecuada en el universo invisible del Espíritu (Wallace, 1889, p. 478).

\section{WALLACE Y SU "NO INFLUENCIA" EN LAS DISCUSIONES MODERNAS}

Los elementos planteados por Wallace en Darwinism son una extensión de los planteados por el propio Darwin, en la medida en que como ya se mencionó, la intención de la obra era aclarar las propuestas presentadas sobre todo en El origen de las especies, pero es claro que sobre todo en temas como la herencia y el caso del ser humano, es la visión muy personal de Wallace la que se impone.

Ahora bien, los elementos defendidos por Wallace en Darwinism, y que conformaron la base del neodarwinismo de finales del siglo XIX, como la importancia absoluta del mecanismo de la selección natural, el concepto biológico de especie, el énfasis en el aspecto utilitarista de las adaptaciones, la divergencia de caracteres, o la aparición de características en los seres humanos como el lenguaje como parte de un proceso cultural $^{9}$, están presentes en formas muy similares dentro de las propuestas que resultaron en la base conceptual de los puntos básicos defendidos por la Síntesis Moderna de la evolución a mediados del siglo $X X$.

Recientemente, con la excusa del centenario del fallecimiento de Wallace, se hicieron recuentos que buscaron valorar el lugar de Wallace dentro de la biología evolutiva moderna (Ruiz, Noguera y Rodríguez, 2015), aunque es claro que el resultado fue que, a pesar de los posibles avances históricos alrededor de la figura y la obra de Wallace, se mantiene largamente ignorado (Kutschera y Hossfeld, 2013; Hossfeld y Osslon, 2013), por cuestiones que parecen ir encaminadas, de manera implícita o explícita, por el "descrédito" generado por sus intereses "extracientificos". Si se hace caso a esto último, se promue- ve así una visión anacrónica de la historia de la ciencia. Wallace, como cualquier otro personaje histórico - o de hecho, como cualquier otra persona - es complejo, y juzgarlo a la distancia a partir de sesgos contemporáneos, es injusto.

Si se parte de las propias evidencias proporcionadas por Wallace en obras como Darwinism, como el concepto de especie biológica, el énfasis en el gradualismo, la importancia de la selección natural por encima de los mecanismos lamarckianos, entre otros, por lo menos queda la impresión de que en su papel de neodarwinista, sentó las bases de buena parte de las discusiones que se dieron alrededor de temas evolutivos hacia finales del siglo XIX y principios del XX. Una reconstrucción histórica no implica remover personajes o teorías, ni validar las propuestas bajo premisas contemporáneas. Por ejemplo, conocer un poco más las ideas de Wallace no le resta ninguna importancia a lo dicho por Darwin, sino por el contrario, se propicia una mejor comprensión de las discusiones científicas en el siglo XIX británico. El punto es buscar las conexiones que se dieron en su momento, $y$, sobre todo, desentrañar la compleja trama en la que se crean las diferentes historias, no solamente de personajes de sobra conocidos, sino de todos aquellos que han contribuido al desarrollo de la ciencia. Wallace, como tantos otros personajes, está todavía por ser descubierto en toda su extensión, en la medida en que, por ahora, tenemos solamente algunos pequeños reflejos de su amplia y diversa labor como naturalista. Sin embargo, no se debe olvidar que en su época a partir de su incansable labor como defensor y promotor de las ideas de Darwin se ganó el título del "primer darwinista", una descripción que podríamos replantear como el "más influyente intérprete del darwinismo".

\section{AGRADECIMIENTOS}

Esta publicación fue posible gracias a la beca posdoctoral del Programa de Becas Posdoctorales de la Dirección General de Personal Académico (DGAPA) de la Universidad Nacional Autónoma de México (UNAM). 


\section{NOTAS}

1. De manera un poco más detallada, estos son los usos que se muestran en el Oxford English Dictionary, segunda edición, 1993.

2. Se puede mencionar también, a partir de una esquela de Darwin publicada en The Times el 21 y el 24 de abril de 1882, que el autor anónimo resalta la pluralidad que entre los hombres de ciencia se habían recibido "el método darwinista y las doctrinas distintivas del darwinismo", lo que refleja esa diversidad en la comprensión de las ideas asociadas con Darwin.

3. Fue en lo privado, en el intercambio de correspondencia que tuvieron en 1890 , en donde se puede apreciar que Romanes llegó a calificar a Wallace como absurdo e incapaz, expresiones que fueron duramente criticadas por el propio Wallace. Romanes aceptó que se extralimitó en sus expresiones y que las suprimió posteriormente, ya que vale la pena aclarar que fueron publicadas en Nineteenth Century. Ver Wallace, 1905, Vol. II, pp. 317-326.

4. Las Gifford Lectures son una serie de conferencias anuales establecidas en 1887 por el juez escocés Adam Gifford (1820-1887), cuyo propósito hasta el día de hoy es promover y difundir el estudio de la teología natural en el más amplio sentido del término.

5. Recientemente, James Costa hace un recuento para mostrar que, solamente en Londres entre 1869 y 1900, se

\section{BIBLIOGRAFÍA}

NOTA: la obra original de Wallace se puede consultar de manera gratuita en línea, a través de: The Alfred Russel Wallace Page, http://people.wku.edu/charles.smith/index1.htm, y Wallace Online, http://wallace-online.org/

Beddall, Barbara G. (1968), "Wallace, Darwin, and the theory of natural selection", Journal of the History of Biology 1(2), pp. 261-323, [en línea]. https://doi.org/10.1007/ BF00351923

Bickerton, Derek (2014), More than Nature Needs, Cambridge y Londres, Harvard University Press.

Bowler, Peter J. (2013), Darwin Deleted: Imagining a World Without Darwin, Chicago y Londres, University of Chicago Press.

Butler, Samuel (1908), Essays on Life, Art and Science, editado por Richard Alexander Streatfield, Londres, A. C. Fifield.

Caponi, Gustavo (2016), “Definitivamente no estaba ahí: la ausencia de la teoría de la selección natural en sobre la tendencia de las variedades a apartarse indefinidamente del tipo original de Alfred Russel Wallace". Ludus Vitalis, 17(32), pp. 55-73.

Costa, James T. (2014), Wallace, Darwin, and the Origin of Species, Cambridge y Londres, Harvard University Press.

Darwin, Charles (1859), On the origin of species by means of natural selection, or the preservation of favoured races in the struggle for life, Londres, John Murray. publicaron por lo menos veinte libros enfocados en el tema. Ver Costa, 2014, p. 271

6. Boston Evening Transcript, Noviembre 2 de 1886, p. 4.

7. La obra biogeográfica de Wallace es sumamente amplia, lo que presentó en Darwinism resumía lo dicho en obras como The Geographical Distribution of Animals; With A Study of the Relations of Living and Extinct Faunas as Elucidating the Past Changes of the Earth's Surface (1876) y Island Life: Or, The Phenomena and Causes of Insular Faunas and Floras, Including a Revision and Attempted Solution of the Problem of Geological Climates (1880).

8. La idea de continuidad como un sinónimo de los procesos evolutivos se debe al físico y juez galés William Robert Grove (1811-1896), quien como presidente de BAAS en 1866, y asesorado por Darwin y Joseph D. Hooker (18171911), presentó por primera vez un discurso evolucionista, como un sinónimo ambiguo de las ideas de Darwin, en una sociedad científica británica. Ver Morus, 2017, p. 129-131.

9. Recientemente, se ha dado en hablar del "problema de Wallace", que busca responder a la pregunta: ¿Cómo adquirió la especie humana una mente que parece mucho más poderosa de lo que los humanos podrían haber necesitado para sobrevivir? Ver Bickerton, 2014, pp. 1-15.

Darwin, Charles; Wallace, Alfred Russel (2009), Selección natural: tres fragmentos para la historia, traducido por Rosaura Ruiz Gutiérrez y Juan Manuel Rodríguez Caso, España, Los Libros de la Catarata.

Desmond, Adrian; Moore, James R. (2009), Darwin, Londres, Penguin Books.

Dixon, Mike; Radick, Gregory (2009), Darwin in Ilkley, Stroud, History Press.

Ellegård, Alvar (1990), Darwin and the General Reader: The Reception of Darwin's Theory of Evolution in the British Periodical Press, 1859-1872, Chicago, University of Chicago Press.

Elsdon-Baker, Fern (2008), "Spirited dispute: the secret split between Wallace and Romanes", Endeavour 32(2), pp. 75-78 [en línea]. https://doi.org/10.1016/j. endeavour.2008.04.008

Fichman, Martin (2004), An Elusive Victorian: The Evolution of Alfred Russel Wallace, Chicago y Londres, University of Chicago Press.

Ginnobili, Santiago; Blanco, Daniel (2019), "Wallace's and Darwin's natural selection theories", Synthese, 196(3), pp. 991-1017 [en línea], https://doi.org/10.1007/ s11229-017-1491-z

Gutjahr, Paul C. (2011), Charles Hodge: Guardian of American Orthodoxy, Oxford y Nueva York, Oxford University Press. 
Henslow, George (1907), "The True Darwinism", Journal of the Royal Horticultural Society, XXXIII, pp. 1-7.

Himmelfarb, Gertrude (1996[1959]), Darwin and the Darwinian Revolution, Chicago, I.R. Dee.

Hodge, Charles (1874), What Is Darwinism?, Nueva York, Scribner, Armstrong, and Company.

Hodge, M. J. S. (1977), "The Structure and Strategy of Darwin's "Long Argument"”, The British Journal for the History of Science 10(3), pp. 237-46 [en línea], https://doi. org/10.1017/S0007087400015685.

Hodge, M. J. S. (2015), "Wallace y Darwin: los dos primeros darwinistas". En Rodríguez, Juan Manuel; Ruiz, Rosaura; Oliver, Celia; Abarca, César (eds.), Alfred Russel Wallace, a cien años de su muerte, México, UNAM, pp. 59-67.

Hossfeld, Uwe; Olsson, Lennart (2013), "The Prominent Absence of Alfred Russel Wallace at the Darwin Anniversaries in Germany in 1909, 1959 and 2009", Theory in Biosciences, 132(4), pp.1-7 [en línea], https://doi.org/10.1007/ s12064-013-0189-0

Hubrecht, Ambrosius; Arnold, Willem (1908), "Darwinism versus Wallaceism", Contemporary Review, 94, pp. 629-634.

Hutton, Frederick Wollaston (1900), "New-Darwinism”, Science, 8(9), pp. 588-589.

Kohn, David (1989) “Darwin's Ambiguity: The Secularization of Biological Meaning", The British Journal for the History of Science, 22(2), pp. 215-39, [en línea], https://doi. org/10.1017/S0007087400026005

Kutschera, Ulrich; Hossfeld, Uwe (2013), “Alfred Russel Wallace (1823-1913): the forgotten co-founder of the Neo-Darwinian theory of biological evolution", Theory in Biosciences, 132(4), pp. 207-214, [en línea] https://doi.org/10.1007/ s12064-013-0187-2

Mallet, James (2009), "Alfred Russel Wallace and the Darwinian Species Concept: His Paper on the Swallowtail Butterflies (Papilionidae) of 1865", Gayana (Concepción), 73, pp. 4254, [en línea] https://doi.org/10.4067/ S0717-65382009000300005

Mayr, Ernst (1991), One Long Argument: Charles Darwin and the Genesis of Modern Evolutionary Thought, Cambridge: Harvard University Press.

Mayr, Ernst (2004), What makes biology unique?: considerations on the autonomy of a scientific discipline, Cambridge, Cambridge University Press.

McKinney, Henry Lewis (1972), Wallace and Natural Selection, New Haven, Yale University Press.

Moore, James (1991), “Deconstructing Darwinism: The politics of evolution in the 1860s", Journal of the History of Biology, 24(3), pp. 353-408 [en línea], https://doi.org/10.1007/ BF00156318

Morus, Iwan Rhys (2017), William Robert Grove: Victorian Gentleman of Science, Aberyswyth, University of Wales Press.
Pleins, J. David (2014), In Praise of Darwin: George Romanes and the Evolution of a Darwinian Believer, Nueva York, Londres, Nueva Delhi, Sydney, Bloomsbury Publishing USA.

Richards, Robert J. (2003), “Biology”. En: Cahan, David (ed.), From Natural Philosophy to the Sciences: Writing the History of Nineteenth-Century Science, Chicago y Londres, University of Chicago Press, pp. 16-48.

Rodríguez Caso, Juan Manuel (2019), “Historización de la naturaleza del ser humano en A. R. Wallace", Saberes. Revista de historia de las ciencias y las humanidades, 2(5), pp. 141-171.

Romanes, George John (1889a), “'Darwinism'”, Nature 40(1044), p. 645. https://doi.org/10.1038/040645a0

Romanes, George John (1889b), "Mr. Wallace on Darwinism", Science 14(343), pp. 150-55.

Rossiter, Margaret W. (1971), "Benjamin Silliman and the Lowell Institute: The Popularization of Science in Nineteenth-Century America", The New England Quarterly 44(4), pp. 60226 [en línea], https://doi.org/10.2307/364476

Ruiz Gutiérrez, Rosaura; Noguera Solano, Ricardo; Rodríguez Caso, Juan Manuel (2015), "La importancia de Alfred R. Wallace en la biología evolutiva". En: Rodríguez, Juan Manuel; Ruiz, Rosaura; Oliver, Celia; Abarca, César (eds.), Alfred Russel Wallace, a cien años de su muerte, México, UNAM, pp. 43-49.

Ruse, Michael (2005), "The Darwinian Revolution, as seen in 1979 and as seen Twenty-Five Years Later in 2004", Journal of the History of Biology, 38(1), pp. 3-17 [en línea], https:// doi.org/10.1007/s10739-004-6506-1

Wallace, Alfred Russel (1865), “I. On the Phenomena of Variation and Geographical Distribution as Illustrated by the Papilionidæ of the Malayan Region", Transactions of the Linnean Society of London, 25(1), pp. 1-71 [en línea], https://doi.org/10.1111/j.1096-3642.1865.tb00178.x

Wallace, Alfred Russel (1870), Contributions to the Theory of Natural Selection: A Series of Essays, Londres y Nueva York, Macmillan \& Co.

Wallace, Alfred Russel (1889), Darwinism: An Exposition of the Theory of Natural Selection, Londres, Macmillan and co.

Wallace, Alfred Russel (1905), My Life: A Record of Events and Opinions (Vols 1-2), Londres, Chapman \& Hall.

Wallace, Alfred Russel (1908), "Darwinism versus Wallaceism”, Contemporary Review, 94, pp. 716-717.

Wallace, Alfred Russel (2013a), Alfred Russel Wallace's 18861887 Travel Diary: The North American Lecture Tour, editado por Charles H. Smith y Megan Derr, Manchester, Siri Scientific Press.

Wallace, Alfred Russel (2013b), On the Organic Law of Change. A Facsimile Edition and Annotated Transcription of Alfred Russel Wallace's Species Notebook of 1855-1859, editado por James T. Costa, Cambridge y Londres, Harvard University Press.

Waters, C. Kenneth (2009), "The arguments in the Origin of Species". En Hodge, M. J. S. y Radick, Gregory (eds.), The Cambridge Companion to Darwin, $2^{\circ}$ ed., Cambridge, Cambridge University Press, pp. 120-143, p. 120-121. 